\title{
Integrating Mathematical Thinking and Creative Problem Solving in Engineering Mathematics Blended Learning
}

\author{
Hamidreza Kashefia ${ }^{*}$, Zaleha Ismail ${ }^{b}$, Yudariah Mohamad Yusof ${ }^{c}$ \\ ${ }^{a}$ Department of Mathematics, Faculty of Science, Kermanshah Branch, Islamic Azad University, Kermanshah, Iran \\ ${ }^{b}$ Department Educational Science, Mathematics and Creative Multimedia, Faculty of Education, University Teknologi Malaysia, 81310 Johor Bahru, \\ Malaysia \\ ${ }^{c}$ Center of Engineering Education, University Teknologi Malaysia, 81310 Johor Bahru, Malaysia \\ *Corresponding author: kashefih@iauksh.ac.ir
}

\begin{abstract}
Engineering students often find their first university multivariable calculus experience very challenging. While moving from single variable function to multivariable function with procedural aspects of the subject, solving limit, derivation, and integral problems, they may struggle with crucial conceptual ideas underpinning them, making it very difficult to progress in more advanced courses. The main objective of this research is to establish a model of teaching and learning by integrating mathematical thinking and creative problem solving to support students in the learning of multivariable calculus through a blended learning environment. The results revealed that blended learning proved a valuable support and adjunct to students' learning.
\end{abstract}

Keywords: Blended Learning; creative problem solving; engineering mathematics; mathematical thinking; multivariable calculus

(C) 2017 Penerbit UTM Press. All rights reserved

\subsection{INTRODUCTION}

Multivariable calculus is one of the most important parts of mathematics syllabus for engineering students. It is offered as a prerequisite course to other advanced mathematics courses and even other courses. However, multivariable calculus is one of the most difficult courses for most engineering students to study in their field (Roselainy, 2009; Kashefi et al., 2010a; Kashefi et al., 2011). Various problematic areas have been identified in basic calculus and multivariable calculus. Some of these were, the difficulty of learning some specific mathematical topics, the difficulty in coordinating procedures and manipulating concepts, the particular events that the students experienced in the past, poor problem solving skills, the inability to select and use appropriate mathematical representations, the translation of real-world problems into calculus formulations, absorbing complex new ideas in a limited time, the students' beliefs and their learning styles (Kashefi et al., 2011).

There have been several attempts to improve engineering students' learning and the teaching of multivariable calculus through moving away from remedial classes towards teaching to increase understanding (Roselainy, 2009). Improving students' learning through the enhancement of their problem solving and mathematical thinking skills as well as through using technological tools to support conceptual understanding and problem solving methods are now thought to be more appropriate. It enables them to cope with the mathematical needed for solving problems in their fields of study.

Researchers that promote mathematical thinking try to support students for the understanding of mathematical concepts and solving real problems in face-to-face classroom. In a study of multivariable calculus for engineering student, Roselainy and her colleagues (Roselainy, 2009; Roselainy et al, 2007a; Roselainy et al, 2007b) presented a model of active learning in face-to-face multivariable calculus classroom. The model was based on invoking students' mathematical thinking powers, supporting mathematical knowledge construction, and promoting generic skills such as communication, teamwork, and self-directed learning, but without using computer. They provided and promoted a learning environment where mathematical powers are used specifically and explicitly towards supporting students (i) to become more aware of the mathematics structures being learned, (ii) to recognize and use their mathematical thinking powers, and (iii) to modify their mathematical learning behaviour.

However, an earlier study using Roselainy et al.'s method found that students still have difficulties when encountering non-routine problems in multivariable calculus, especially functions of two variables (Kashefi et al., 2010a; Kashefi et al., 2011). Some student obstacles are: (i) sketching the graph of two-variable functions in 3-dimensions, (ii) confusion in the use of symbols in representing twovariable functions, (iii) students' idiosyncrasies attributed from previous mathematical construction, (iv) inability to select appropriate representation of mathematics worlds, (v) transition from one world to other world of mathematics. The findings confirmed the need and the importance of a new environment to support students' mathematical knowledge construction and to help students overcome their obstacles in multivariable calculus.

In this study, blended learning has been proposed as an appropriate environment to promote mathematical thinking (Kashefi et al., 2009; Kashefi et al, 2012). Although some work has been done and reported on modelling mathematical thinking in face-to-face multivariable 
calculus classes, the literature review indicates that very few studies have been carried out or reported on the integration of strategies to invoke mathematical thinking explicitly in a blended learning environment. The main purpose of this study is to establish a blended learning model that conceptualizes a framework for supporting mathematical knowledge construction and invokes students' mathematical thinking powers and skills. Subsequently the study determines the impact of this environment on the students learning of multivariable calculus and in overcoming students' obstacles.

\subsection{BLENDED LEARNING MATHEMITICS MODEL}

Tall (Tall, 2004) based on the theory of three modes of representation of human knowledge (Bruner, 1966) point out that there are not only three distinct types of mathematics worlds; there are in fact three significantly different worlds of mathematical thinking: conceptualembodied, proceptual-symbolic, axiomatic-formal. Tall (Tall, 1989; Tall, 1993) refined the theory of building and testing conceptual structures of Skemp (Skemp, 1979) and found that the computer provides an environment and that give a new way for building and testing mathematical concept by supporting all three modes of building and testing conceptual structures in Skemp theory. In fact, the computer brings a new dimension into the "didactic triangle" model including pupil, teacher, and mathematics in the face-to-face learning environment. According to Tall (Tall, 1989; Tall, 1986), there are now four components with new role and relation among the student, the teacher, the mathematics, and computer as forming tetrahedral in a suitable learning environment.

In fact, the Tall's theory of three worlds of mathematical thinking underlies the creation of computer software which Tall called generic organizer and used it in his researches (Tall, 2000; Tall, 2003) to support students' mathematical construction and build embodied approach to mathematical concepts. In designing the generic organizer, it requires the selection of an important foundational idea to focus on. Tall used the notion of cognitive root as a cognitive unit containing the seeds of cognitive expansion to formal definitions and later theoretical development. Tall showed how the notion of local straightness (for rate of change/differentiation) and area under the graph (for cumulative growth/integration) can be cognitive roots in building an embodied understanding of the calculus. However, the generic organiser does not guarantee the understanding of the concept and Tall (Tall, 1993; Tall, 1997) reported some cognitive obstacles faced by students when using this organiser. Tall believed that the learner requires an external organising agent in the shape of guidance from a teacher, textbook, or some other agency. In this way, Tall suggested that the combination of a human teacher and a computer environment can support students' mathematical knowledge construction and prevent misleading factors.

In the study of multivariable calculus, Tall's theory insists on a flexible blend of embodiment and symbolism. As for the transition from one variable to two, two variables form one vector variable and the idea of local straightness becomes local flatness and the locally straight approach that is based on a blend of embodiment and formalism. However, Tall researches more focus on supporting students' mathematical thinking powers to construct mathematical content in basic calculus. Tall (Tall, 1997) endeavored to explain the construction of mathematical concepts in Basic Calculus. He focused on students' difficulties and used computers as a way of supporting students' mathematical thinking to overcome these difficulties (Tall, 1993).

The definition of blended learning as a combination of F2F formats and web-based formats identified an environment that includes two important components of Tall's method: a generic organizer (computer) and an organizing agent (teacher) (Kashefi et al., 2012). In fact, the blended learning environment has rich tools to extend Tall's approach in promoting mathematical thinking in multivariable calculus. Furthermore, the use of e-learning as an important element of blended learning provides sufficient tools to support students' generic skills. It is proposed that blended learning has the potential to improve Roselainy et al.'s model for supporting students in three aspects of learning.

There is very little in the literature to identify the components that are involved in the learning process of blended learning. According to Tall (Tall, 1986), computers bring a new dimension into the 'didactic triangle' model. Albano (Albano, 2005) extended the 'didactic triangle' model and gave the four vertices as the author, the teacher, the student and knowledge, with new roles and relations assigned to each, thereby changing the learning process in e-learning environments. Norazah and her colleagues (Norazah et al., 2005), also based on Henry (Henry, 2001), hypothesized that the key elements of e-learning solutions are content, technology, service and strategy. Combining the main components of Tall (Tall, 1986) and Albano (Albano, 2005), the author, teacher, student, strategy, technology, and mathematics can be hypothesized as the components of blended learning (Kashefi et al., 2010b).

In the didactic scheme in blended learning, the author as the creator of the mathematics course has to define and identify the role of each component. The relationship between students, the relationship between students and teacher, and the relationship of both with other components are the most important aspects of the didactic scheme in blended learning. The author, who can also be a teacher, prepares mathematics tasks and assessments using technology tools and pedagogical strategies such as communication, teamwork and problem solving. In fact, mathematics, technology and strategy are important recourses in designing a blended learning mathematics course (Kashefi et al., 2012). A blended learning environment will give students the opportunity to benefit from both F2F and e-learning instruction. In this environment, the theoretical foundation on which the development of strategies for mathematical knowledge construction and the enhancement of students' mathematical thinking are based on the work of Tall (Tall, 2004, Tall, 2007). Frameworks from Watson and Mason (Watson \& Mason, 1998) and the works of Lumsdaine and Lumsdaine (1995) were used to design classroom activities and tasks. The following aspects are given taking into consideration the development and implementation of blended learning in multivariable calculus courses (Kashefi et al., 2012; Kashefi et al., 2013).

(i) Classroom tasks- in designing the tasks, the existing tasks based on the preliminary study are selected and modified to focus the students' attention on the mathematical knowledge construction, thinking processes and generic skills through blended learning. This is done by implementing various mathematical thinking powers, strategies and tools that are used in preparing the tasks as the most important elements of a website recourse that are designed for F2F class and laboratory session as online and offline. All mathematical tasks used in the classroom are compiled as website that is divided in different sections.

(ii) Assessments- understanding of mathematical facts, standard methods and techniques, standard application of concepts, problem solving, and logical and analytical reasoning are mostly the difficulties that the students face in the learning of multivariable calculus. These aspects are the objects of assessments (Roselainy, 2007). In this method, we used both summative and formative types of assessments that include quizzes and tests, quick classroom feedback and written assignment in F2F and online formats. 
(iii) Computer and web aide- by using the web environment, the resources are prepared in the most sufficient ways for it to be used in F2F class and in laboratory session (as online \& offline). It will also help the students in their study at home. In this environment, students can have access to lecture notes, web-based interactive educational tools, animations, videos, forums module, chat module, journal module, assignments, assessments, survey and feedback. In addition, it will also help the students to find more information about content and questions, and to submit assignments, projects and laboratory reports.

(iv) Strategies- designing prompts and questions are being used in order to initiate mathematical communication between the students and lecturer. Furthermore, synchronous and asynchronous web communication facilities such as chat, email, and discussion board can also support the students' oral and written communication. Moreover, by doing group assignments and presentations as a team, not only they can support the students' team work but they can also encourage discussion and sharing of ideas among the students. Working in pairs, small group, critical thinking and problem solving, students' own examples, doing assignments, reading and writing in the F2F and web environment are other strategies of this method. Figure 1 shows the model of blended learning mathematics which is used as a guide to classroom instruction.

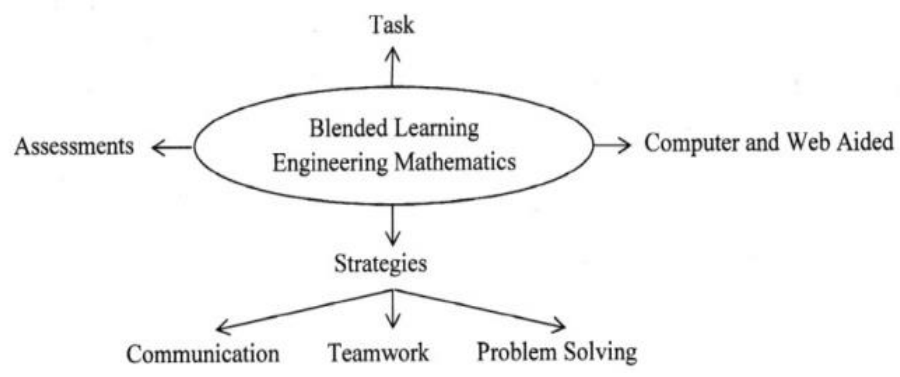

Figure 1 Block diagram of the processes of the system

Technology (computer and web aided) as an element of blended learning model, that includes tools such as files, video, discussion forums, chat, journal, animation, and test's systems, can support didactical methods such as lectures, discussion, assessment that are used to teach mathematics. Thus, technology as a part of blended learning model can also support the students' thinking and generic skills. In other words, the designing of tasks, assessments, and web resources in a special manner based on the strategies can be supported by technology based on mathematical thinking and CPS. Specifically, students' generic skills can be supported by using sufficient tools in elearning as an important element of blended learning model.

\subsection{THE STUDY}

Process

One class of 62 first year students among 154 engineering students that enrolled in a multivariable calculus was selected randomly for this study at Islamic Azad University of Kermanshah (IAUKSH) in Iran. The students comprised of 48 males and 14 females aged 18 to 21 in Civil Engineering, Computer Engineering, Electrical Engineering, and Industrial Engineering fields. The multivariable calculus offered by IAUKSH is a three credit undergraduate course and covers functions of several variables, partial derivatives, multiple integrals, vector functions and vector calculus. These topics were taught over a period of 14 weeks with 3 meeting hours per week consisting of 2 hours face-to-face and a 1 hour laboratory session. In the lecture session, the mathematical concepts were introduced to the whole class. After the students had established a general idea of the concept, they then proceeded to the laboratory session. In the laboratory session, online activities, students were directed to perform interactive mathematics tasks, and to post messages and questions on the discussion board. The instructional design of the textbook was based on researchers' methodology to inculcate mathematical thinking and CPS. Thus, we designed prompts and questions based on mathematical thinking and CPS to increase the students' understanding, teamwork, and communication by organizing the contents in the specified manner. In addition, a course discussion board was used to foster studentstudent and student-instructor communication during the course providing a collaborative and shared space for a global community.

\section{Sample}

Observing the students during the semester in the F2F and the laboratory sessions and the median $(M d=175.50)$ of their activities based on the web course reports helped the instructor to divide the students into two groups. Then, six students from each group were chosen randomly as upper and lower groups in using the web course and the laboratory facilities. 
Table 1 The upper group students

\begin{tabular}{lccc}
\hline Students Name & View & All Changes & All Activities \\
\hline Amin & 489 & 94 & 583 \\
Hadis & 496 & 29 & 525 \\
Ali H & 437 & 57 & 494 \\
Sanaz & 260 & 53 & 313 \\
Tooraj & 161 & 50 & 211 \\
Salar A & 145 & 40 & 185 \\
\hline
\end{tabular}

Table 1 represents the students in the upper group and the records of their actions on the web course including the numbers of view and all changes such as add, update, delete and course enroll. Table 2 shows the name of the lower group students.

Table 2 The lower group students

\begin{tabular}{lccc}
\hline Students Name & View & All Changes & All Activities \\
\hline Saman & 136 & 33 & 169 \\
Keyvan & 96 & 45 & 141 \\
Mostafa & 71 & 44 & 115 \\
Ahmad & 86 & 23 & 109 \\
Yazdan & 43 & 27 & 70 \\
Tahereh & 2 & 1 & 3 \\
\hline
\end{tabular}

\section{Instruments}

The methods of data collection employed for this study was qualitative in nature, which includes participants' think-aloud verbalizations and task-based interview. Data for the study were collected based on a "one-to-one" basis where each participant solved every problem under direct observation of the researcher in an enclosed room arranged by the school authority. Every participant was instructed to thinkaloud and show all their solutions on the space provided in the instrument while solving every problem. After solving each problem, the participant was interviewed to collect supplementary verbal data via retrospective questioning. Audio tapes were used to collect their verbal protocols while video tapes were used to record the whole process of their written solutions. The audio recording of the verbal protocols was converted into verbatim transcripts, while the visual recording of the participants' written solution processes was generated into descriptive texts. The typewritten transcripts and texts were then given to the individual participants for verification. Any changes due to disagreement of words or phrases used in the transcripts or texts were made based on negotiations of both the participants and the researchers.

To ensure a clear account in elucidating students' mathematical thinking process and in identifying the effectiveness of the course on students' learning of multivariable calculus concepts, think-aloud protocol was conducted. Using verbal protocol could help further in making explicit the solution strategies and the explanation of the solution processes and answers beside the identification of the participants' mathematical thinking and CPS. The task-based interview was used to collect supplementary verbal data on the participants' solution strategies. It involved retrospective questioning to supplement think-aloud verbalization.

Since the participants were required to verbalize while solving the problems, the problems chosen must enable the participants to find it easy to think aloud (Bryne, 1983). In relation to that, the problems must be well specified and did not demand very abstract reasoning and long chains of deduction. Five problems were prepared where students had to solve 4 problems without using computers and 1 problem is solved using computers. The 4 problems to be solved via think-aloud verbalizations without using computer (Problems $1,2,3$, and 4) and 1 problem to be solving using computers (Problem 5) were as follows.

1. Find the domain and range for the function: $f(y, z)=\sqrt{64-4 y^{2}-z^{2}}$ and sketch the domain of $\mathrm{f}$.

Problem 1 involves finding the domain and the range of a function not only in terms of $\mathrm{y}$ and $\mathrm{z}$ but also under the radical which is not the same with other popular function such as $f(x, y)=\sqrt{a-x^{2}-y^{2}}$.

2. Sketch the graph of the surfaces of $y=x^{2}$ and $x^{2}-y^{2}=1$.

Problem 2 includes equations to test on the students' ability in sketching the graphs of cylinders in $\mathbb{R}^{3}$. The first equation $z=y^{2}$ was a cylinder in terms of different variables that students solved in the class. The aim of the second equation $x^{2}-y^{2}=1$ was to show how students could extend their knowledge in sketching the graph of a cylinder that was not discussed in the class.

3. If $z=u^{2}-v$ where $u=\tan (x+y)$ and $v=\frac{x}{y}$, find $\frac{\partial z}{\partial x}$ in terms of $\mathrm{x}$ and $\mathrm{y}$ by using the Chain Rule.

Problem 3 involved finding the derivatives of a two-variable function. The first aim of the problem was to find out whether the students were able to write the formula if the $\mathrm{z}$ is a function of $\mathrm{u}$ and $\mathrm{v}$ instead of $\mathrm{x}$ and $\mathrm{y}$. Another aim of this problem was to show how the students combine ideas based on the Chain Rules with partial derivatives. Furthermore, in the task-based interview the differences between $\frac{\partial z}{\partial x}$ and $\frac{d z}{d x}$ were asked from the students. 
4. Given the integral $\int_{0}^{1} \int_{\frac{1}{4}}^{1} f(x, y) d y d x+\int_{1}^{2} \int_{\frac{1}{4}}^{\frac{1}{x^{2}}} f(x, y) d y d x$

(a) Sketch the region of integration.

(b) Write down the limits of integration if the order of integration is change to $d x d y$.

Problem 4 involved sketching the region of integration in order to find the limits of integration if the order of integration is changed from $d y d x$ to $d x d y$. The first aim of the problem was to determine whether the students were able to sketch the region of integration based on the limits of both double integrals. Then, to show how the students combine both regions and to find the limit of integration if the order of integration is changed from $d y / d x$ to $d x / d y$.

5. Explore the results of the transformation $g_{1}(x, y)=c f(x, y)$ and $g_{2}(x, y)=f\left(c_{1} x, c_{2} y\right)$ graphically.

Problem 5 involves the description of what changes will occur for the graph of $f(x, y)$ when the constants are added as $c f(x, y)$ and $f\left(c_{1} x, c_{2} y\right)$. In fact, the students had to sketch the graph of the $g_{1}(x, y)=c f(x, y)$ and $g_{2}(x, y)=f\left(c_{1} x, c_{2} y\right)$.

Field work starts when the students are met individually. Students need to access to computer and web-based tools in order to solve the fifth problem. Firstly, the researcher will have to explain on the think aloud procedures and demonstrate the process of verbalizing while thinking. After the explanations as well as the demonstration are done, students will solve the problems. The researcher carried out the data collection process in separate sessions for each of the problem involving different days.

Script is not necessary for the follow-up questions but the researcher need to ask questions based on the events that happened during the think-aloud protocol. For example, when a student fails to fully verbalize, the researcher may ask a process question ("How did you solve that?") or when a student spend several minutes on a particular problem, the researcher may ask a design question ("Was there anything that confused you?").

There were three sources of data - verbatim transcripts and descriptive texts. These two sources of data were then organized according to each problem for every participant. Based on the participants' written solutions, the researchers described and then classified the participants' errors. The corresponding transcripts were then referred to look for the related verbal protocols that seemed to provide evidence of where, how or why the errors happened.

Analysis of participants' errors is presented based on each problem. Participants "e written solutions which had been optically scanned are included in this paper to show the errors made by them. Wherever appropriate, participants' verbal protocols during thinking-aloud or interview were quoted as support to the discussion of where, how or why the errors were made. For ethical reasons, the names used in this paper are pseudonyms of the participants.

\section{Data Analysis}

Data analysis begins with the transcription of all the think-aloud verbal protocols to generate individual verbatim reports based on students' solutions that are obtained either by using or without using computer. All the task-based interviews protocols are also transcribed into written dialogue. According to Ericsson and Simon (Ericson and Simon, 1993), it is important to carefully produce precise transcripts of the recorded tapes after the collection of verbal data, thus protecting the raw data in as 'hard' a form as could be wished. This approach allows the data to be clearly analyzed through coding, which is subjectivity in coding the data, since it eliminates problems related to 'soft' data.

Payne (Payne, 1994) stated that by breaking up the protocols into short segments or phrases is an important approach to coding the data that are produced by using think-aloud protocols. In this method, each phrase should refer to what constitutes as a single task assertion or reference by the subject. These segments can then be coded and analyzed. Similarly, Keys (Keys, 2000) suggested that the data are transcribed in full and then break up into clause-length segments. As a result, through coding and categorizing in such way, the researcher is able to identify the types of thinking evident in the think-aloud data.

A coding scheme was developed to code the verbalizations using mathematical thinking and CPS's five steps problem solving process based on Lumsdaine and Lumsdaine (Ericson and Simon, 1993). The mathematical activities can be represented by students in the different steps of CPS that notes in the Table 3.

Table 3 The coding sheet based on CPS steps and mathematical thinking activities

\begin{tabular}{llc}
\hline \multicolumn{1}{c}{ CPS Steps } & \multicolumn{1}{c}{ Mathematical Thinking Activities } & Code \\
\hline \multirow{2}{*}{ Problem Definition } & Exemplifying & DE \\
& Specializing & DS \\
Idea Generation & Conjecturing & GC1 \\
& Completing & GC2 \\
& Deleting & ED \\
& Correcting & EC1 \\
Idea Evaluation & Comparing & EC2 \\
& Sorting & ES \\
& Organizing & EO \\
& Explaining & JE \\
& Justifying & JJ \\
Idea Judgment & Verifying & JV \\
& Convincing & JC \\
& Refuting & JR \\
Implementation of Ideas & Generalizing & IG \\
\hline
\end{tabular}


These codes reflect the though process of students while problem solving. After coding each student's solution for all problems, a rubric was adapted based on the action associated with mathematical thinking powers (Watson \& Mason, 1998) and the McDougall and Karadag rubric (McDougai, 2008) as shown in Table 4.

Table 4 Action associated with mathematical thinking powers

\begin{tabular}{|c|c|c|}
\hline CPS Steps & Mathematical Thinking & Ability/Action \\
\hline \multirow[t]{2}{*}{ Problem Definition } & Exemplifying & $\begin{array}{l}\text { Recognise the specific features or attributes that } \\
\text { makes an object or concept as an example }\end{array}$ \\
\hline & Specializing & Construct special examples to see what happens \\
\hline \multirow[t]{2}{*}{ Idea Generation } & Conjecturing & $\begin{array}{l}\text { Stating a general form but whose truth is not yet } \\
\text { established }\end{array}$ \\
\hline & Completing & Know what to add \\
\hline \multirow{5}{*}{ Idea Evaluation } & Deleting & To remove or to alter to allow \\
\hline & Correcting & To ensure or to contradict between expressions \\
\hline & Comparing & $\begin{array}{l}\text { Recognise similar attributes/properties as well as the } \\
\text { different ones }\end{array}$ \\
\hline & Sorting & Can sort according to certain criteria \\
\hline & Organizing & Can organise according to certain criteria \\
\hline \multirow{5}{*}{ Idea Judgment } & Explaining & Elucidate \\
\hline & Justifying & $\begin{array}{l}\text { Revealing an underlying structure/relationships that } \\
\text { links }\end{array}$ \\
\hline & Verifying & Checking the relationships \\
\hline & Convincing & $\begin{array}{l}\text { Ensuring your reasoning are plausible, convince } \\
\text { yourself, a friend then an enemy }\end{array}$ \\
\hline & Refuting & Looking for contradictions \\
\hline Implementation of Ideas & Generalizing & $\begin{array}{l}\text { Detect a form or general pattern, moving from a few } \\
\text { instances to making guesses about a wide class of } \\
\text { cases }\end{array}$ \\
\hline
\end{tabular}

The qualitative data analysis was completed by the analysis of students' written solutions processes to assessments, semi-structured interview, students' web comments such as social forums and journal module, and semi-structured questionnaire. The PASW 18 was used to analyze the quantitative part of the data.

The framework shows the implementation of TVE as an independent variable, teaching methods, instructional and infrastructural facilities, teaching materials, machines and equipment, as well as learning skills as intervening variables while technical skills, entrepreneurship skills and employability skills are the dependent variables. The background study for the conceptual framework on skills for employment growth and development for this study examines the skill development in order to achieve greater productivity and employment growth. Quality technical and vocational education provides a strategic link to higher productivity, employment and development. What is particularly important to note for the purpose of this study is that productivity at individual level is affected by such factors as education, training, core skills and experience. Absence of relevant and affordable training programs impedes skills development and employability. Thus, the road to more productive employment is impaired.

\subsection{RESULTS}

The analysis of data is discussed based on each participants and problems.

\section{Analysis based on Students}

In this section, the analysis of data is represented based on the overview of each student of the upper and the lower groups on the modes of representation and the solution strategies that they used in solving all five problems based on mathematical thinking and CPS.

\section{Upper Group Students}

During the data collection sessions, Amin was able to think-aloud for all problems. He managed to solve all problems successfully. Analysis of Amin's written solutions revealed his consistency in using the symbolic and embodied worlds of mathematics. For instance, he sketched the graph of domain in Problem 1 in 3-dimansions. In fact, Amin was able to sketch the graph of domain by using the appropriate world of mathematics (here the symbolic world) and the transition from the symbolic world to the embodied world of mathematics. Amin's verbal protocols suggested his ability in making use of common similarity and difference in Problem 5 by using computer tools through CPS's steps. Specializing, sketching, symbolizing, conjecturing, completing, explaining, justifying, and generalizing were inferred in almost all problems.

Hadis was one of the students in this study who could solve most of the problems successfully but failed to find the range in Problem 1 , unable to sketch the second graph in Problem 2, and also failed in solving Problem 4. She was able to give think-aloud verbal protocols for the problems during the data collection sessions; however, task-based interview was necessary to collect her retrospective verbal protocols for these problems and also for Problem 3.

Analysis of Hadis's written solutions suggested that the usage of multiple presentations of mathematics worlds especially the embodied world was demonstrated in almost all of the problems. Furthermore, in problems that involve finding the range, partial 
derivative, and sketching the transformations of graph, she justified her solutions based on the graph. In finding the domain in Problem 1, she did not simplify $4 y^{2}+z^{2} \leq 64$ as $\frac{y^{2}}{16}+\frac{z^{2}}{64} \leq 1$ and she was also unable to find the range because of her poor prior knowledge. In addition, Hadis did not sketch the second graph in Problem 2. Moreover, her difficulty in identifying the region of integration in Problem 4 was related to her lack of ability in transferring from the symbolic world of mathematics (the formula of the integral) to the embodied world of mathematics (the region of integration). Hadis's difficulties in solving these problems were associated with the concepts and techniques. Inference from Hadis's protocols suggested that various strategies of mathematical thinking underlie her solution process. Specializing, sketching, symbolizing, conjecturing, deleting, correcting, justifying, and generalizing were used in almost all problems. Hadis was able to use computer tools to solve Problem 5 through the CPS's steps.

During the data collection sessions, Ali $\mathrm{H}$ was able to think-aloud and to solve all problems successfully. The retrospection in the form of task-based interview was conducted in order to obtain more information about his solutions processes in solving Problems 3 and 5. Analysis of his written solutions suggested that he used multiple presentations of mathematics worlds. Ali $\mathrm{H}$ was able to sketch the graph of domain in 3-dimansions by choosing the appropriate world of mathematics (the symbolic world) and the transition from the symbolic world to the embodied world of mathematics. The use of one mode of representation could be seen in his written solutions for Problem 2 , where he used the embodied representation of mathematics world. Ali H's could solve Problem 5 by using computer tools through the CPS's steps; however, it was only based on one example. Specializing, sketching, symbolizing, conjecturing, completing, explaining, justifying, and generalizing were the mathematical thinking activities that could be inferred in almost all problems.

During the data collection sessions, Sanaz was able to think-aloud for almost all of the problems except for Problem 1 and 4. Therefore, retrospection in the form of task-based interview was necessary for Problems 1, 3, and 4 to supplement her thinking-aloud protocols. Sanaz was able to solve almost all problems successfully except for Problem 1 that required her to find the domain and the range of $\mathrm{f}$ in and Problem 4 on the lack combination of two double integrals in the final step of solving.

Analysis of her written solution suggested she had utilized the embodied and the symbolic representation of mathematics worlds. Her ability to use multiple representations was proven in Problems 2, 3, and 4(a), which was choosing the appropriate world of mathematics and the transition from one world to other world. However, the lack of written communication may be the reason of her inability in correctly writing the domain of Problem 1 . Her difficulty in finding the range in this problem and sketching the graph in Problem 4(a) were the difficulty associated with the concepts. For the problems, the graphs became the base of her justifications. Analysis of Sanaz's verbal protocols suggested that she could use the process of CPS in solving Problem 5 by using computer tools. Inference of Sanaz's verbal protocols suggested that mathematical thinking strategies such as specializing, sketching, conjecturing, justifying, and generalizing were dominant in her solutions of the problems.

Tooraj was not able to give complete think-aloud protocols for all problems during the data collection sessions. Therefore, retrospection in the form of task-based interview was necessary to collect supplementary verbal data for all problems in order to find more information on his solutions processes. Tooraj managed to solve most of the problems successfully except for Problems 2 and 4.

Analysis of his written solutions suggested that he had applied the multiple representations of mathematics worlds in Problem 1 as choosing the appropriate world of mathematics and transition from one world to another world. In finding the domain in Problem 1, Tooraj did not simplify $4 y^{2}+z^{2} \leq 64$ as $\frac{y^{2}}{16}+\frac{z^{2}}{64} \leq 1$ and he had also sketched the second graph in Problem 2 in the wrong orientation. Because of his poor ability in the symbolic world of mathematics, Tooraj could not combine the two double integrals in Problem 4 . His difficulties in solving these problems were associated with the concepts and technique.

Analysis of Tooraj's verbal protocols revealed various bases of his justifications. He justified his solutions based on the graph for Problems 1 and 5. The Mathematical thinking that was dominant from his solution processes in solving problems includes "the similarity" and "the difference" in Problems 5. In addition, specializing, sketching, conjecturing, completing, explaining, and justifying were inferred in solving problems. Tooraj also used computer tools to solve Problem 5 based on the CPS process.

Salar A was able to give a complete think-aloud verbal protocol for Problems 1, 3, and 5 in the data collection sessions. In the cases of when his thinking-aloud protocols were incomplete or when more information was needed to analyzed his solutions processes, retrospection in the form of task-based interview became necessary for Problems 2, 3, and 4. Salar A managed to solve Problems 2 and 4 correctly.

Analysis of Salar A's written solutions suggested that multiple presentations were used in all problems. However, he could not sketch the second graph in Problem 2 and he also had difficulty in combining two double integrals in Problem 4 due to his poor ability in the symbolic world of mathematics. Salar A's difficulties were conceptual. Analysis of his verbal protocols revealed that he used the CPS process in solving Problem 5. He justified Problem 5 based on his graph down. Inference on Salar A's verbalizations suggested that by using prompts and questions and also mathematical thinking strategies such as specializing, sketching, symbolizing, conjecturing, completing, explaining, justifying, and generalizing could infer his solution process in solving the problems.

\section{Lower Group Students}

During the data collection, Saman was not able to think-aloud for the problems. Therefore, retrospection in the form of task-based interview was conducted in order to understand how he solved all problems. Saman only managed to find the domain and sketched it in Problems 1, he also sketched the first graph of Problem 2, and Problem 3. However, he could not solve Problems 4 and 5 at all.

Multiple representations of mathematics worlds were only used in Problem 1 and sketching the first graph of Problem 2. In solving Problem 1, Saman found the domain correctly by using the appropriate world of mathematics (here is the symbolic world). He was not able to simplify the statement, but he could sketch the graph of the domain correctly by using the appropriate transition from symbolic world to embodied world. Poor algebraic manipulation was the reason of his difficulties in finding the range. Saman was able to write the formula of the Chain Rule and solve this problem correctly even without sketching a root diagram for Problem 3 . He also did not know the differences between $\partial u / \partial x$ and $d u / d x$. Saman's difficulties in solving these problems were related to the concepts and the studying of mathematics. Moreover, mathematical thinking strategies such as specializing and conjecturing were inferred based on his protocols. 
Keyvan's thinking-aloud was available for Problems 1, 2, and 3. Thus, retrospection in the form of task-based interview was conducted in order to understand how he tried to solve Problems 4 and 5 and to understand his knowledge in Problems 1 and 3 . Keyvan managed to solve Problems 1, 2, and 3 .

Analysis of his written solutions suggested that he used multiple representations of mathematics worlds in solving the problems. However, Keyvan's justification of the differences between $\partial z / \partial x$ and $d z / d x$ was based on the symbolic world of mathematics. He explained that the difference of $\partial z / \partial x$ and $d z / d x$ is due to $\partial z / \partial x$ is the partial derivative of a two-variable function of $\mathrm{z}$ in respect to $\mathrm{x}$, while $d z / d x$ is derivative of a single variable function in respect to $\mathrm{x}$. Keyvan was one of the students that sketched the graph and wrote the limits of integration by the transition from the symbolic world of mathematics (the formula of integration) to the embodied world of mathematics (the graph). He was able to find the limits of integration when $d y d x$ was changed to $d x d y$ based on the graph (the transition from the embodied world to the symbolic world of mathematics). However, Keyvan wrote the integral as two multiple integrals. Keyvan tried to use the embodied world of mathematics as a sufficient world for solving Problem 5. Nevertheless, he could not find the correct answer because of his inability in finding the transition from the problem equation (the symbolic world) to the embodied world. Keyvan's difficulties appeared to be associated with the concepts and the studying of mathematics. Inference of Keyvan's verbal protocols led to the identification of mathematical thinking embedded in his solution process. Sketching, conjecturing, and justifying were inferred in solving the problems.

Mostafa was able to think-aloud for Problems 1 and 3. Therefore, retrospection in the form of task-based interview was needed for all problems to supplement the data from his thinking-aloud verbalization. Mostafa only managed to solve Problem 1 and 3.

Mostafa used multiple representations of mathematics worlds in solving Problems 1. To solve Problem 2, Mostafa chose the embodied world of mathematics as the appropriate mathematics world. However, his lack of understanding of the concept had led him to sketch both graphs in two dimensions. Although Mostafa could solve Problem 3, he did not know the differences between $\partial z / \partial x$ and $d z / d x$ in Problem 3. Mostafa did not provide an answer to Problems 4 and 5 at all. His difficulties were with the concepts and the studying of mathematics and based on his protocols, only conjecturing, deleting, and correcting as mathematical thinking strategies were inferred.

During the data collection, Ahmad was not able to think-aloud. Thus, retrospection in the form of task-based interview was conducted in order to understand how he solved all problems. Ahmad only managed to solve the first and the second parts of Problems 1 and he did not respond to Problems 4 and 5 at all.

Ahmad used multiple representations of mathematics worlds to solve Problem 1. His lack of understanding of the concept caused him to sketch both graphs in Problem 2 in two dimensions. He also sketched the second graph in a wrong orientation because of his poor prior mathematical knowledge. Ahmad could not remember the Chain Rule formula and furthermore, he wrote the formula for Problem 3 incorrectly. Ahmad also did not know the differences between $\partial u / \partial x$ and $d u / d x$. Ahmad did not answer Problems 4 and 5 at all and his difficulties in solving these problems were associated with concepts, techniques, and the studying of mathematics. Ahmad's justification was based on the symbolic world of mathematics. Only specializing, conjecturing, deleting, and correcting as mathematical thinking activities could be inferred through his protocols.

Yazdan was able to think-aloud for all problems except for Problems 5. The retrospection in the form of task-based interview was conducted in order to understand how he solved Problems 1, 3, and 5 and his underlying thought processes. Yazdan was able to solve all problems except for Problem 5.

Analysis of Yazdan's written solutions suggested that he used multiple representations of the mathematics worlds for all of the problems except for Problem 5. For example, in solving Problem 1, Yazdan used the symbolic world as an appropriate world of mathematics to find the domain. By the transition from the symbolic world to the embodied world, he could sketch the graph of the domain correctly. Yazdan's inability in using computer tools prevented him from implementing any CPS strategies in solving Problem 5. In fact, he tried to use the embodied world of mathematics as a sufficient world for solving the problem; however, the transition from the problem equation (the symbolic world) to the embodied world was not correct. Yazdan's difficulties in solving these appealed problems seemed to be with the concepts and with the studying of mathematics. Mathematical thinking strategies such as sketching, symbolizing, conjecturing, and justifying in solving Problems 2, 3, and 4 were inferred.

During the data collection, Tahereh could hardly think-aloud. So, retrospection in the form of task-based interview was conducted in order to understand how she solved the problems and her underlying processes of thought. Tahereh only managed to solve Problem 3. However, in solving this problem she could not find the correct answer in the final step.

Analysis over Tahereh's solutions suggested that because of poor algebraic manipulation, she could not find the domain of function in Problem 1. Furthermore, poor prior knowledge and inability in the transition from the symbolic world of mathematics to the embodied world caused her to sketch the graph of domain in this problem as circle. Tahereh used the multiple representations of mathematics in Problem 1 which was the embodied and symbolic worlds of mathematics representations. Her lack of understanding of the concept had caused Tahereh to not be able to sketch the first graph of Problem 2 and because of her poor background knowledge, she sketched $x^{2}-$ $y^{2}=1$ in yz-plane incorrectly. Tahereh was able to find the Chain Rule formula by choosing the appropriate world of mathematics (the embodied world), by drawing a label tree diagramed and by transition to the symbolic world of mathematics. Tahereh did not know the differences between $\partial z / \partial x$ and $d z / d x$. Tahereh could not sketch the regions of integrations for Problem 4 correctly. However, she could combine the limits of both integrals based on the symbolic world of mathematics. Tahereh used the embodied world of mathematics as a sufficient world for solving Problem 5. However, the answer that she provided was incorrect due to her inability in the transition from the symbolic world to the embodied world. Tahereh's difficulties appealed seem to be with the concepts, technique, and studying of mathematics. Based on her protocols, only conjecturing as a mathematical thinking strategy in problems was not inferred.

\section{Analysis based on Problems}

This section shows the findings pertaining to the upper and the lower group students and their difficulties in solving the problems. In the analysis of each problem, the results of the analysis for both upper and lower groups of students are discussed separately. 


\section{Problem 1}

\section{Results Analysis of Analysis for Upper Group Students}

In finding the domain, three students (Amin, Ali H, \& Salar A) could solve the problem correctly. Two students (Hadis \& Tooraj) found the domain as: $D_{f}=\left\{(y, z) \mid 4 y^{2}+z^{2} \leq 64\right\}$ and sketched it as ellipse; however, Hadis could not find the range correctly. Sanaz was able to sketch the graph of domain, but she was unable to find the domain and range correctly.

To present the findings related to this problem, the solution process from three students (Amin, Hadis, \& Sanaz) were used. Ali H and Salar A's solution process were similar to Amin's. The transcription of Amin's verbal protocol during solving Problem 1 was as follows. In this problem, first we want to find the domain and the range and then we want to sketch the graph of domain. Because the function is a square root function, the quantity under square root cannot be negative. Then, we will have: $64-4 y^{2}-z^{2} \geq 0$. Now we simplify the equation [... calculation]. So we have: $4 y^{2}+z^{2} \leq 64$. Because the coefficients of $\mathrm{y}$ and $\mathrm{z}$ in the equation are not equal, thus, the equation does not represent a circle. By dividing both side of equation by 64 we have: $\frac{y^{2}}{16}+\frac{z^{2}}{64} \leq 1$ which is an ellipse. According to the definition of domain for two-variable function, the domain of a twovariable function is all $(y, z)$ that $\frac{y^{2}}{16}+\frac{z^{2}}{64} \leq 1$. So, the domain is as: $D_{f}=\left\{(y, z) \mid \frac{y^{2}}{16}+\frac{z^{2}}{64} \leq 1\right\}$. For finding the range, we have to find the output of the function. Because the function is a square root function so the output cannot be negative. If $y=$ $z=0$ then $f(0,0)=8$, which is the maximum value for $\mathrm{f}$. For some points such as $(4,0)$ on the ellipse we have the minimum value for $\mathrm{f}$ that is 0 . So the range is[0,8]. For sketching the graph of domain, the equation is in terms of $\mathrm{y}$ and $\mathrm{z}$, thus, we have to sketch the domain on yz-plane. Since the biggest denominator is under z, thus the ellipse is vertical [sketching].

Figure 2 represents Amin's written solution for Problem 1.

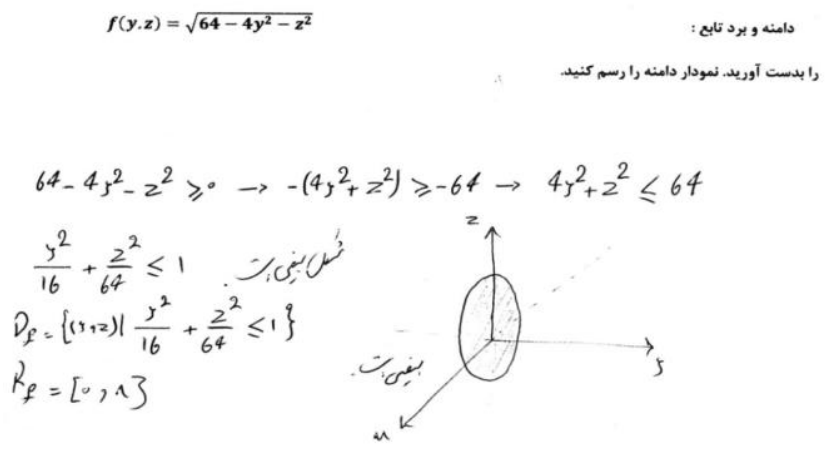

Figure 2 Amin's written solution for Problem 1

Hadis was one of the students who found the domain as $D_{f}=\left\{(y, z) \mid 4 y^{2}+z^{2} \leq 64\right\}$ and sketched it correctly. She could also find the range correctly. Her transcript is shown below.

[Reading the problem twice] First we have to find the domain. Since the function is the square root, the quantity under the radical is greater than 0 or equal to 0 . So, we have: $64-4 y^{2}-z^{2} \geq 0$. [calculation] By simplifying of this equation we have: $4 y^{2}+z^{2} \leq 64$. Then the domain is as: $D_{f}=\left\{x, y \mid 4 y^{2}+z^{2} \leq 64\right\}$. Because the function is in terms of $\mathrm{y}$ and $\mathrm{z}$, thus we have to sketch the domain in yz-plane. The coefficients of the equation are not equal so the equation presents an ellipse. Now we have to find the range. The function is the square root function $f(y, z)$ cannot be negative; therefore, the range is $[0,+\infty)$. It needs to be explained that if $-4 y^{2}-z^{2}$ is greater than 64 , then the quantity under the radical will be negative. So, $y$ and $z$ must be greater than 64 . If $-4 y^{2}-z^{2}$ is equal to 0 , then the $\mathrm{f}$ is 0 .

Table 5 represents the results of the upper group students' responses to Problem 1.

Table 5 The upper group students' results in solving Problem 1

\begin{tabular}{lcccccc}
\hline \multicolumn{1}{c}{ Problem 1 } & Amin & Hadis & Ali H & Sanaz & Tooraj & Salar A \\
\hline (a) Finding the domain & $\sqrt{ }$ & $\sqrt{ }$ & $\sqrt{ }$ & - & $\sqrt{ }$ & $\sqrt{ }$ \\
(b) Finding the range & $\sqrt{ }$ & $\sqrt{ }$ & $\sqrt{ }$ & $\sqrt{ }$ & $\sqrt{ }$ & $\sqrt{ }$ \\
(c) Sketching the domain & $\sqrt{ }$ & - & $\sqrt{ }$ & - & $\sqrt{ }$ & $\sqrt{ }$ \\
\hline
\end{tabular}

\section{Results Analysis of Analysis for Lower Group Students}

Three out of the six students (Keyvan, Mostafa, \& Yazdan) managed to get the correct answer. Saman and Ahmad were the students that wrote the domain as: $\left\{(y, z) \mid 4 y^{2}+z^{2} \leq 64\right\}$ and sketched it correctly. However, they could not find the range correctly. Tahereh showed inability in algebraic manipulation in finding the domain. She sketched the graph of domain as a circle of radius 2.

Tahereh was a student that was not able to solve the problem correctly. Her verbal transcripts show her solution process. 
[reading the problem] For finding the domain we have: $64-4 y^{2}-z^{2} \geq 0$ [calculation]. So, we have: $4 y^{2}+z^{2} \leq-64$ that represents a circle with radius of 8 . Then, we can write the domain as: $D_{f}=\left\{(y, z) \mid 4 y^{2}+z^{2} \leq-64\right\}$.

The graph of domain is a circle of radius 8 . The range is ... [silent] ... [sorry] ...

To illustrate why she could not sketch the domain and did not find the range, the interview session with Tahereh was quoted.

Researcher: Why did you sketch the graph as a circle?

Tahereh: $\quad$ Because this statement [points to her response] is the formula of circle ...

Researcher: $\quad$ Do you know the formula of a circle?

Tahereh: $\quad \ldots$ Yes, $x^{2}+y^{2}=r^{2} \ldots$

Researcher: In this problem the coefficient of $x^{2}$ is 4 . Can you explain?

Tahereh: $\quad \quad$... Yes ... We can write it as $(2 x)^{2} \ldots$

Researcher: Why did you not find the range?

Tahereh: $\quad$... Sorry I have difficulty in finding the range ...

Figure 3 shows Tahereh's written solution for Problem 1.

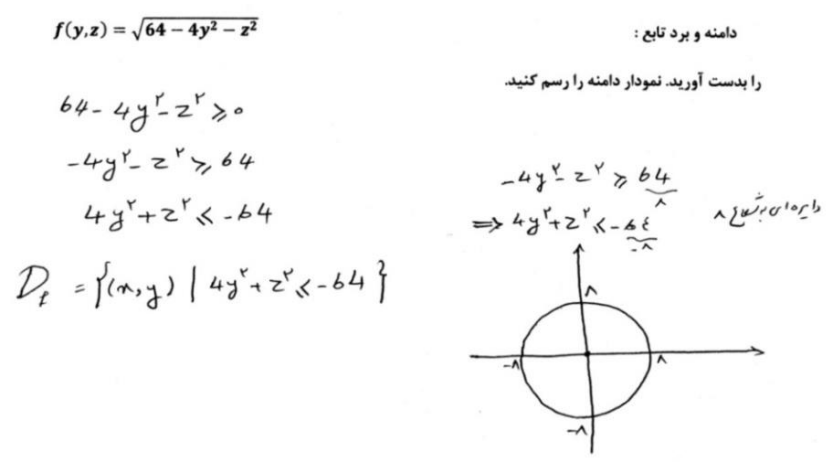

Figure 3 Tahereh's written solution for Problem 1

Table 6 represents the results of the upper group students' responses to Problem 1.

Table 6 The Lower group students' results in solving Problem 1

\begin{tabular}{lcccccc}
\hline Problem 1 & Saman & Keyvan & Mostafa & Ahmad & Yazdan & Tahereh \\
\hline (a) Finding the domain & $\sqrt{ }$ & $\sqrt{ }$ & $\sqrt{ }$ & $\sqrt{ }$ & $\sqrt{ }$ & - \\
(b) Finding the range & $\sqrt{ }$ & $\sqrt{ }$ & $\sqrt{ }$ & $\sqrt{ }$ & $\sqrt{ }$ & - \\
(c) Sketching the domain & - & $\sqrt{ }$ & $\sqrt{ }$ & - & $\sqrt{ }$ & - \\
\hline
\end{tabular}

\section{Problem 2}

\section{Results Analysis of Analysis for Upper Group Students}

Three out of six students (Ali H, Amin, \& Sanaz) were able to solve this problem correctly. Two students (Salar A \& Hadis) could only sketch the graph of $z=y^{2}$. Tooraj was also a student that not only could not sketch the graph of $x^{2}-y^{2}=1$. but also sketched the graph of $z=y^{2}$ in a wrong orientation. Due to many similarities of the solution's processes, only the processes of solution from three students (Ali H, Salar A, \& Tooraj) are presented.

Ali H, Amin, and Sanaz were able to solve the problem by using the traces of the surface. However, most of these students did not write any symbols and calculation to find the traces and they explained it verbally.

Table 7 represents the results of the upper group students' responses in solving Problem 2.

Table 7 The upper group students' results in solving Problem 2

\begin{tabular}{lcccccc}
\hline Problem 2 & Amin & Hadis & Ali H & Sanaz & Tooraj & Salar A \\
\hline (a) Sketching $\boldsymbol{z}=\boldsymbol{y}^{\mathbf{2}}$ & $\sqrt{ }$ & $\sqrt{ }$ & $\sqrt{ }$ & $\sqrt{ }$ & - & $\sqrt{ }$ \\
(b) Sketching $\boldsymbol{x}^{\mathbf{2}}-\boldsymbol{y}^{\mathbf{2}}=\mathbf{1}$ & $\sqrt{ }$ & - & $\sqrt{ }$ & $\sqrt{ }$ & - & - \\
\hline
\end{tabular}

\section{Results Analysis of Analysis for Lower Group Students}

Two of the students (Keyvan \& Yazdan) were able to solve this problem successfully. Another two students (Saman \& Tahereh) could only sketch the graph of $z=y^{2}$. Mostafa and Ahmad sketched the graph of cylinders as graph of single- variable functions in $\mathbb{R}^{3}$ incorrectly. Mostaf's solution process is presented. The solution processes of Keyvan and Yazdan's solutions were similar to Ali H's 
solution from the upper group, and Saman and Tahereh's solution processes were also similar to Salar A's from the upper group, thus their solution processes are not presented.

Table 8 represents the results of the lower group students' responses to Problem 2.

Table 8 The Lower group students' results in solving Problem 2

\begin{tabular}{lcccccc}
\hline Problem 2 & Saman & Keyvan & Mostafa & Ahmad & Yazdan & Tahereh \\
\hline (a) Sketching $\boldsymbol{z}=\boldsymbol{y}^{\mathbf{2}}$ & $\sqrt{ }$ & $\sqrt{ }$ & - & - & $\sqrt{ }$ & $\sqrt{ }$ \\
(b) Sketching $\boldsymbol{x}^{\mathbf{2}}-\boldsymbol{y}^{\mathbf{2}=\mathbf{1}}$ & - & $\sqrt{ }$ & - & - & $\sqrt{ }$ & - \\
\hline
\end{tabular}

\section{Problem 3}

\section{Results Analysis of Analysis for Upper Group Students}

All six students (Sanaz, Amin, Hadis, Ali H, Tooraj, \& Salar A) could compute $\partial z / \partial x$ completely. They drew a label tree diagram to show the relationships among the variables. To present the findings related to this problem, Sanaz's solution process is used. Other five (Amin, Hadis, Ali H, Tooraj, \& Salar A) students's written solutions were similar to Sanaz's.

All students in their interview knew the differences between $\partial z / \partial x$ and $d z / d x$. However, in the interview, Hadis and Salar A only explained that $\partial z / \partial x$ is the partial derivative of a two-variable function of $\mathrm{z}$ in respect to $\mathrm{x}$ and $d z / d x$ is derivative of a single-variable function in respect to $\mathrm{x}$.

Table 9 represents the results of the upper group students' responses in solving Problem 3.

Table 9 The upper group students' results in solving Problem 3

\begin{tabular}{lcccccc}
\hline Problem 3 & Amin & Hadis & Ali H & Sanaz & Tooraj & Salar A \\
\hline Finding $\frac{\partial z}{\partial y}$ & $\sqrt{ }$ & $\sqrt{ }$ & $\sqrt{ }$ & $\sqrt{ }$ & $\sqrt{ }$ & $\sqrt{ }$ \\
\hline
\end{tabular}

\section{Results Analysis of Analysis for Lower Group Students}

Two students (Keyvan \& Yazdan) were able to solve this problem successfully. These students could write the formula based on that evaluated $\partial z / \partial x$ correctly by drawing a tree diagram. Saman and Mostafa did not draw the tree diagram for solving this problem; however, they were also successful in answering solving the problem. Two students (Tahereh and Ahmad) also used a labeled tree diagram to find the Chain Rule formula. However, they could not solve this problem correctly. Ahmad was also one of the students that could not solve Problem 3.

Table 10 represents the results of the lower group students' responses to Problem 3.

Table 10 The Lower group students' results in solving Problem 3

\begin{tabular}{lcccccc}
\hline Problem 3 & Saman & Keyvan & Mostafa & Ahmad & Yazdan & Tahereh \\
\hline Finding $\frac{\partial z}{\partial y}$ & $\sqrt{ }$ & $\sqrt{ }$ & $\sqrt{ }$ & - & $\sqrt{ }$ & - \\
\hline
\end{tabular}

\section{Problem 4}

\section{Results Analysis of Analysis for Upper Group Students}

Two students (Amin \& Ali H) were able to solve this problem correctly. Sanaz, Tooraj, and Salar A were the students that could sketch the region and found the limits correctly; however, in the last step, they did not combine to integral as a double integral. Hadis was a student that could not solve this problem. Due to the similarity of the processes of solution, only Salar A and Hadis's solution processes are presented. Salar A was a student that did not combine both regions in order to write them as a double integral. Hadis was one of the students that were unable to solve the problem correctly.

Table 11 represents the results of the upper group students' responses in solving Problem 4.

Table 11 The upper group students' results in solving Problem 4

\begin{tabular}{lcccccc}
\hline Problem 4 & Amin & Hadis & Ali H & Sanaz & Tooraj & Salar A \\
\hline Part (a) & $\sqrt{ }$ & - & $\sqrt{ }$ & $\sqrt{ }$ & $\sqrt{ }$ & $\sqrt{ }$ \\
Part (b) & $\sqrt{ }$ & - & $\sqrt{ }$ & $\sqrt{ }$ & $\sqrt{ }$ & $\sqrt{ }$ \\
\hline
\end{tabular}




\section{Results Analysis of Analysis for Lower Group Students}

Yazdan was the only student that was able to solve this problem correctly. Two students (Keyvan \& Tahereh) also were able to write the integral as two double integrals; however, they could not combine both integrals as an integral. Saman, Mostafa, and Ahmad were the students that could not solve this problem at all. The solution process of Yazdan was similar to Amin's and Ali H's solutions, and Keyvan and Tahereh's solution proceses were similar to Salar A's. Keyvan wrote the integral as two multiple integrals

Table 12 represents the results of the lower group students' responses to Problem 4.

Table 12 The Lower group students' results in solving Problem 4

\begin{tabular}{lcccccc}
\hline Problem 4 & Saman & Keyvan & Mostafa & Ahmad & Yazdan & Tahereh \\
\hline Part (a) & - & $\sqrt{ }$ & - & - & $\sqrt{ }$ & $\sqrt{ }$ \\
Part (b) & - & $\sqrt{ }$ & - & - & $\sqrt{ }$ & $\sqrt{ }$ \\
\hline
\end{tabular}

\section{Problem 5}

\section{Results Analysis of Analysis for Upper Group Students}

Students in the upper group respond to this problem by using computer and accessing to the web course. All students could show what happened to the graph of $f(x, y)$ by adding the constants as $c f(x, y)$ and $f\left(c_{1} x, c_{2} y\right)$ by using the Multivariable Calculus Online tools (Krisley, 2001) to sketch the graphs. Four of the students (Amin, Hadis, Sanaz, \& Salar A) could explain the graphical transformations based on the five steps of CPS. Two students (Ali H \& Tooraj) were also able to solve and explain the transformation, but their conjecture and justification were based on only one example. It means that in the first step of CPS, they chose and tested only one example and based on the changes of the graphs they immediately responded to the problem. These students in the interview noted that because they were able to guess the solution of the problem, they only checked that for one c.

Table 13 represents the results of the upper group students' responses in solving Problem 5.

Table 13 The upper group students' results in solving Problem 5

\begin{tabular}{lcccccc}
\hline Problem 5 & Amin & Hadis & Ali H & Sanaz & Tooraj & Salar A \\
\hline Part (a) & $\sqrt{ }$ & $\sqrt{ }$ & $\sqrt{ }$ & $\sqrt{ }$ & $\sqrt{ }$ & $\sqrt{ }$ \\
Part (b) & $\sqrt{ }$ & $\sqrt{ }$ & $\sqrt{ }$ & $\sqrt{ }$ & $\sqrt{ }$ & $\sqrt{ }$ \\
\hline
\end{tabular}

\section{Results Analysis of Analysis for Lower Group Students}

Students in this group also used a computer that was connected to the Internet in order to solve Problem 5. At the beginning, all students did not want to use computer tools. However, after the lack of success in solving the problem, two (Saman \& Ahmad) students out of six requested to use the computer tools. The instructor helped them to access to the Multivariable Calculus Online tools (Krisley, 2001) via the web course. However, after sketching the graph of $x^{2}+y^{2}$ that was the default of online tool they could not type the equations such as $2\left(x^{2}+y^{2}\right)$ as $2 *\left((x)^{\wedge} 2+(y)^{\wedge} 2\right)$ because of their lack of experience in using computer tools.

Three students (Keyvan, Mostafa, \& Taraneh) could not respond to the problem at all while only one student (Yazdan) tried to solve the problem manually.

Table 14 represents the results of the lower group students' responses to Problem 5.

Table 14 The Lower group students' results in solving Problem 5

\begin{tabular}{lcccccc}
\hline Problem 5 & Saman & Keyvan & Mostafa & Ahmad & Yazdan & Tahereh \\
\hline Part (a) & - & - & - & - & - & - \\
Part (b) & - & - & - & - & - & - \\
\hline
\end{tabular}

\subsection{DISCUSSION}

Results of upper group students' verbal protocols revealed that specializing, conjecturing, completing, explaining, justifying, and generalizing were the common mathematical thinking activities that occurred during solving the think-aloud problems. Most students used multiple representations of mathematics worlds, especially the symbolic and the embodied worlds, in solving the problems. Table 15 represents the students' mathematical thinking activities through the CPS steps of the problems for 6 students in the upper group Group students' mathematical thinking activities through CPS steps of problems. 
Table 15 Upper Group students' mathematical thinking activities through CPS steps of problems

\begin{tabular}{|c|c|c|c|c|c|c|c|c|c|c|c|}
\hline \multirow[t]{2}{*}{ CPS Steps } & \multirow[t]{2}{*}{ Code } & \multicolumn{3}{|c|}{$\mathrm{P} 1$} & \multicolumn{2}{|c|}{$\mathrm{P} 2$} & \multirow[t]{2}{*}{ P 3} & \multicolumn{2}{|c|}{$\mathrm{P} 4$} & \multicolumn{2}{|c|}{ P 5} \\
\hline & & (a) & (b) & (c) & (a) & (b) & & (a) & (b) & (a) & (b) \\
\hline \multirow[t]{2}{*}{ Problem Definition } & $\mathrm{DE}$ & 6 & & & & & & & & 5 & 6 \\
\hline & DS & 6 & 1 & & 5 & 3 & 6 & 6 & & 6 & 6 \\
\hline \multirow[t]{2}{*}{ Idea Generation } & GC1 & 6 & 6 & 6 & 6 & & 6 & 5 & 6 & 6 & 6 \\
\hline & GC2 & 5 & 3 & 4 & & & 5 & 5 & 4 & 6 & 6 \\
\hline \multirow[t]{5}{*}{ Idea Evaluation } & ED & & & & & 1 & & & & & \\
\hline & EC1 & & & 4 & & 1 & & & & & \\
\hline & $\mathrm{EC} 2$ & 5 & 5 & & & & & & & 6 & 6 \\
\hline & ES & & & & & & & & & 6 & 6 \\
\hline & EO & & & & & & & & & 6 & 6 \\
\hline \multirow[t]{5}{*}{ Idea Judgment } & $\mathrm{JE}$ & 5 & & 4 & & 3 & 6 & & & 6 & 6 \\
\hline & JJ & 5 & 6 & 4 & & 3 & 6 & 5 & & 6 & 6 \\
\hline & JV & & 1 & 4 & & 3 & 6 & & & & \\
\hline & $\mathrm{JC}$ & & 1 & 4 & & & 6 & & & & \\
\hline & JR & 2 & & & & & & & & & \\
\hline Implementation of Ideas & IG & & 5 & & 5 & 3 & 6 & 5 & & 6 & 6 \\
\hline Stops without solution & & 1 & & & & & & 1 & 2 & & \\
\hline Makes errors & & & & 2 & 1 & & & 1 & 1 & & \\
\hline $\begin{array}{l}\text { Significance inability to } \\
\text { problem }\end{array}$ & & & & & & 3 & & & & & \\
\hline
\end{tabular}

Based on the verbal protocols of the lower group students, it can be seen that conjecturing was the highest mathematical thinking activities that occurred during the solving of the think-aloud problems. In addition, specializing and justifying could also be seen among the students when they were solving the think-aloud problems. Some students, in solving the problems, used the symbolic and the embodied worlds of mathematics as multiple representation of mathematics. The students' appealed difficulties in solving problems were with concepts and techniques.

Table 16 Lower Group students' mathematical thinking activities through CPS steps of problems

\begin{tabular}{|c|c|c|c|c|c|c|c|c|c|c|c|}
\hline \multirow[t]{2}{*}{ CPS Steps } & \multirow[t]{2}{*}{ Code } & \multicolumn{3}{|c|}{ P1 } & \multicolumn{2}{|c|}{ P 2} & \multirow[t]{2}{*}{ P 3} & \multicolumn{2}{|c|}{ P 4} & \multicolumn{2}{|c|}{ P5 } \\
\hline & & (a) & (b) & (c) & (a) & (b) & & (a) & (b) & (a) & (b) \\
\hline \multirow[t]{2}{*}{ Problem Definition } & DE & 2 & 2 & & & & & & & 2 & 2 \\
\hline & DS & 2 & 2 & & 6 & 5 & 2 & 3 & & 2 & 2 \\
\hline \multirow[t]{2}{*}{ Idea Generation } & $\mathrm{GC} 1$ & 6 & 6 & 5 & 6 & 2 & 6 & 3 & 3 & & 1 \\
\hline & GC2 & 2 & 3 & & & & & 3 & 1 & & \\
\hline \multirow[t]{5}{*}{ Idea Evaluation } & ED & 2 & 2 & 1 & & & & & & & \\
\hline & $\mathrm{EC} 1$ & 2 & 2 & 1 & & & 1 & & & & \\
\hline & $\mathrm{EC} 2$ & & & & & & & & & & \\
\hline & ES & & & & & & & & & & \\
\hline & EO & & & & & & & & & & \\
\hline \multirow{5}{*}{ Idea Judgment } & $\mathrm{JE}$ & & 5 & & & 2 & 2 & & & & \\
\hline & $\mathrm{JJ}$ & & 5 & 4 & & 2 & 5 & 3 & & & \\
\hline & $\mathrm{JV}$ & & 2 & & & 2 & 2 & & & & \\
\hline & $\mathrm{JC}$ & & 2 & & & & 2 & & & & \\
\hline & $\mathrm{JR}$ & & & & & & & & & & \\
\hline Implementation of Ideas & IG & & 2 & & 4 & 2 & & 3 & & & \\
\hline \multicolumn{2}{|l|}{ Stops without solution } & & & 1 & & & & & & & \\
\hline \multicolumn{2}{|l|}{ Makes errors } & 1 & 1 & 2 & 2 & 2 & 2 & & & & 1 \\
\hline \multicolumn{2}{|c|}{$\begin{array}{l}\text { Significance inability to solve } \\
\text { problem }\end{array}$} & & & & & 2 & & 3 & 3 & 4 & 3 \\
\hline
\end{tabular}

Table 16 shows the students' mathematical thinking activities for 6 students in the lower group during the problem solving process. Table 17 represents the types of students' difficulties and the numbers of correct answers made by the upper and the lower groups of students. Most students in the upper group could solve the problems correctly. The second part of Problem 2 was seen as the most difficult problem among the upper group students and only three students (50\%) could solve this problem correctly. The second part of Problem 1, Problem 3, and Problem 5 were the problem that all upper group students could solve correctly. For the lower group students, Problem 5 was the most difficult problem and no students could solve it correctly. Moreover, only two students (33\%) in this group solved the second part of Problem 2. Most students in this group could solve the first and second parts of Problem 1. The inability to sketch and poor prior knowledge on the concepts were the reasons of students' difficulties in solving problems. These difficulties were associated with concepts, techniques, and with the studying of mathematics. 
Table 17 Comparison of the upper and lower groups' students' abilities and their difficulties in solving the problems

\begin{tabular}{|c|c|c|c|c|c|}
\hline \multirow[b]{2}{*}{ Problems } & & \multicolumn{2}{|c|}{ Upper Group Students } & \multicolumn{2}{|c|}{ Lower Group Students } \\
\hline & & $\begin{array}{l}\text { Correct } \\
\text { Answer }\end{array}$ & Difficulty & $\begin{array}{l}\text { Correct } \\
\text { Answer }\end{array}$ & Difficulty \\
\hline Problem 1 & (a) & 5 & $\begin{array}{l}\text { Poor writing } \\
\text { communication }\end{array}$ & 5 & Algebraic manipulation \\
\hline & (b) & 6 & & 5 & Sketching \\
\hline & (c) & 4 & Poor prior knowledge & 3 & $\begin{array}{l}\text { - Lack of knowledge } \\
\text { (1) } \\
\text { - Poor prior knowledge } \\
\text { (2) }\end{array}$ \\
\hline Problem 2 & (a) & 5 & Sketching & 4 & Sketching \\
\hline & (b) & 3 & Sketching & 2 & Sketching \\
\hline Problem 3 & & 6 & & 4 & $\begin{array}{l}\text { - Lack of knowledge } \\
\text { - Poor technique for } \\
\text { derivation }\end{array}$ \\
\hline Problem 4 & (a) & 6 & & 3 & \\
\hline & (b) & 5 & Finding the region & 3 & Lack of knowledge \\
\hline Problem 5 & (a) & 6 & & 0 & Sketching \\
\hline & (b) & 6 & & 0 & Sketching \\
\hline
\end{tabular}

\subsection{CONCLUSION}

The understanding of the effectiveness of blended learning multivariable calculus course on students' learning and problem solving skills involve looking into the students' processes of solving problems. In this study, the students' strategies of solutions and modes of representation led to the inference on how the blended learning multivariable calculus course affected the students' mathematical knowledge construction, problem solving, and reduction of their difficulties.

The proposed method was successful in addressing not only the students' concern in getting good examination results but also in enhancing their mathematical thinking ability by using sufficient mathematical activities and tasks based on the design of the model. The findings obtained seem to support the theoretical framework of this study. When the students use different mathematical thinking strategies such as specializing, conjecturing, completing, explaining, justifying, and generalizing in solving different problems that all of them worked out through oral and written prompts and questions, represent the effect of the blended learning multivariable calculus course. Furthermore, when the students use different modes of representation clarify that the blended learning multivariable calculus course supports the symbolic and the embodied worlds of mathematics or even sometimes the formal world of mathematics. It seems that designing prompts and questions to support and develop both symbolic and embodied worlds of mathematical thinking, help students in choosing the appropriate world of mathematics and the transition from one world to other world of mathematics, which is seen as the two important reasons of students' difficulties in multivariable calculus based on the findings of the preliminary investigation.

Solving problems based on the multiple processes of CPS could also be seen in the students' solutions. Adopting CPS helped students in solving problems by invoking communication and teamwork skills through technology tools. Using computer tools also helped students in solving the problems that are difficult to be solved by only depending on mathematical thinking and CPS strategies.

Most students showed their ability to overcome the difficulties in solving problems that was reported in the preliminary investigation. Students' solutions to written assessments and interviews revealed the effect of the blended learning multivariable calculus course in reducing the students' difficulties. However, algebraic manipulation and poor prior knowledge related to basic calculus concepts are the reasons of why some students still have difficulties in solving problems (Kashefi, Zaleha, Yudariah, \& Roselainy, 2012b). Students' comments also confirmed the effectiveness of the course on their learning.

\section{References}

Albano, G. (2005). Mathematics and e-Learning: A Conceptual Framework, DIDIA-Center of Excellence "Methods and Systems for Learning and Knowledge", University of Salerno, Italy.

Bruner, J. S. (1966). Towards a Theory of Instruction, New York: Norton.

Ericsson, K. A., and Simon, H. A. (1993). Protocol Analysis: Verbal Reports As Data (rev.ed.), Cambridge, MA: The MIT Pres.

Henry, H. (2001). E-learning Technology, content and Services, Education + Training, Volume 43, Number 4.

Kashefi, H., Zaleha Ismail., and Yudariah Mohd Yusof. (2010a). Obstacles in the Learning of Two-variable Functions through Mathematical Thinking Approach, Procedia - Social and Behavioral Sciences 8, pp. 173-180.

Kashefi, H., Zaleha Ismail., and Yudariah Mohd Yusof. (2010b). Engineering Mathematics Obstacles and Improvement: A Comparative study of Students and Lecturers Approaches through Creative Problem Solving, In CD Proceedings of the 3rd Regional Conference on Engineering Education \& Research in Higher Education, Kuching, 7-9 Jun.

Kashefi, H., Zaleha Ismail., and Yudariah Mohd Yusof. (2009). Promoting Mathematical Thinking in Engineering Mathematics through Blended E-learning, Proceedings of the Education Postgraduate Research Seminar, November 18-19, Johor Bahru, Malaysia, 34-46.

Kashefi, H., Zaleha Ismail., and Yudariah Mohd Yusof. (2011). Students' Difficulties in Multivariable Calculus Through Mathematical Thinking Approach, Journal of Edupres, 1, 77-86.

Kashefi, H., Zaleha Ismail., Yudariah Mohd Yusof, and Roselainy Abd. Rahman. (2012a). Fostering Mathematical Thinking in the Learning of Multivariable Calculus through Computer-Based Tools, 4th World Conference on Educational Sciences, WCES 2012, A Paper Accepted to be published in Procedia - Social and Behavioral Sciences.X

Kashefi, H., Zaleha Ismail., and Yudariah Mohd Yusof. (2012). Using Blended Learning to Support Students Thinking Powers in Multivariable Calculus, Jurnal Teknologi, 57 (Sains Sosial),. 1-14. 
Kashefi, H., Zaleha Ismail., Yudariah Mohd Yusof., and Mirzaei, F. (2013). Generic Skills in Engineering Mathematics through Blended Learning: A Mathematical Thinking Approach, International Journal of Engineering Education, 9(5),. 1222-1237.

Keys, C. W. (2000). Investigating The Thinking Processes Of Eighth Grade Writers During The Composition Of A Scientific Laboratory Report, Journal of Research in Science Teaching, 37(7), 676-690.

Knisley, J. (2001). Multivariable Calculus Online, Retrieved March 13, 2010, from http://math.etsu.edu/multicalc/prealpha/contents.htm

Lumsdaine, M., and Lumsdaine, E. (1995). CPS Thinking Skills for A Changing World, Edward, McGraw-Hill.

McDougall, D., and Karadag. Z. (2008). Tracking Students' Mathematical Thinking Online: Frame Analysis Method, 11th International Congress on Mathematical Education. Monterrey, Nuevo Leon, Mexico.

Norazah Mohd Nordin., Halimah Badioze Zaman., and Rosseni Din. (2005). Integrating Pedagogy and Instructional Design in the e-Learning Approach for the Teaching of Mathematics, Proceedings of the Second International Conference on e-Learning for Knowledge-Based Society, August 4-7.

Payne, J. W. (1994). Thinking Aloud: Insights into Information Processing. Psychological Science, 5(5), 241-248.

Roselainy Abd. Rahman., Yudariah Mohd Yusof., and Mason, J. H. (2007a). Translating Learning Theories into Practise in Enhancing Students' Mathematical Learning at University, Proceding of the Third International Conference on Research and Education on Mathematics.

Roselainy Abd. Rahman., Sabariah Baharun., and Yudariah Mohammad Yusof. (2007b). Enhancing Thinking through Active Learning in Engineering Mathematics, In CD Proceedings of Fourth Regional Conf. on Engineering Educ., Johor Bahru, 3-5 Dec.

Roselainy Abd. Rahman. (2009). Changing My Own and My Students Attitudes Towards Calculus Through Working on Mathematical Thinking, Unpublished PhD Thesis, Open University, UK.

Skemp, R. R. (1979). Intelligence, Learning, and Action: A Foundation For Theory And Practice In Education, Chichester: John Wiley.

Tall, D. O. (1986). Using The Computer As An Environment For Building And Testing Mathematical Concepts: A Tribute To Richard Skemp, in Papers in Honour of Richard Skemp, 21-36, Warwick.

Tall, D. O. (1989). Concept Images, Generic Organizers, Computers \& Curriculum Change, For the Learning of Mathematics, 9(3), 37-42.

Tall, D. O. (1993). Computer environments for the learning of mathematics, Didactics of Mathematics as a Scientific Discipline- The State of the Art, ed R. Biehler, R.

Tall, D. O. (1997). Functions and Calculus. In A. J. Bishop et al. (Eds.), International Handbook of Mathematics Education, 289-325, Dordrecht: Kluwer.

Tall, D. O. (2000). Biological Brain, Mathematical Mind and Computational Computers, Plenary Presentation for ATCM Conference, Chang Mai, Thailand.

Tall, D. O. (2003). Using Technology to Support an Embodied Approach to Learning Concepts in Mathematics, First Coloquio de Historia e Tecnologia no Ensino de Matemática, at Universidade do Estado do Rio De Janeiro, February 21-3, 2002,1-28

Tall, D. O. (2004). Thinking Through Three Worlds of Mathematics. Proceedings of the 28th Conference of the International Group for the Psychology of Mathematics Education, Bergen, Norway, 4, 281-288.

Tall, D. O. (2007). Embodiment, Symbolism and Formalism in Undergraduate Mathematics Education, Plenary at 10th Conference of the Special Interest Group of the Mathematical Association of America on Research in Undergraduate Mathematics Education, Feb 22-27, San Diego, California, USA.

Watson, A., and Mason, J. (1998). Questions and Prompts for Mathematical Thinking, AMT, Derby. 